\title{
Discrete Grüss type inequality on fractional calculus
}

\author{
Elvan Akin', Serkan Aslıyüce², Ayşe Feza Güvenilir ${ }^{2 *}$ and Billur Kaymakçalan ${ }^{3}$
}

${ }^{\text {*Correspondence: }}$

guvenili@science.ankara.edu.tr

2Department of Mathematics,

Faculty of Science, Ankara

University, Ankara, Turkey

Full list of author information is

available at the end of the article

\author{
Abstract \\ We give a discrete Grüss type inequality on fractional calculus. \\ MSC: Primary 39A12; 34A25; 26A33; secondary 26D15; 26D20 \\ Keywords: discrete fractional calculus; discrete Grüss inequality
}

\section{Introduction}

Motivated by Grüss [1], our purpose is to prove more general versions of Grüss type inequalities for delta discrete fractional calculus. It is well known that Grüss type inequalities in continuous and discrete cases play a crucial role in studying the qualitative behavior of differential and difference equations, respectively, as well as many other areas of mathematics [2-9]. For the background and a summary on these particular subjects, we refer the interested reader to the excellent references [2, 10-18].

The study of discrete fractional calculus was pioneered by Diaz and Osler [19]. In the mentioned work, the authors used an infinite sum to give a definition of discrete fractional sum, whereas Gray and Zhang used a finite sum in [20]. In the last decade, new results in this area have been established [21-24], as well as importance has been gained by inequalities on discrete fractional calculus in [10, 24-27]

\section{Preliminaries}

We begin with basic definitions and results from [10].

Definition 1 The $v$ th fractional sum of $f$ is defined by

$$
\Delta^{-v} f(t, a)=\frac{1}{\Gamma(v)} \sum_{s=a}^{t-v}(t-s-1)^{\frac{v-1}{}} f(s)
$$

where $f$ and $\Delta^{-v} f$ are defined for $s=a \bmod (1)$ and $t=(a+v) \bmod (1)$, respectively. In particular, $\Delta^{-v}$ maps functions defined on $\mathbb{N}_{a}$ to functions defined on $\mathbb{N}_{a+v}$, where $\mathbb{N}_{t}=$ $\{t, t+1, t+2, \ldots\}$.

Here,

$$
t^{\underline{v}}:=\frac{\Gamma(t+1)}{\Gamma(t-v+1)} .
$$

From now on in this context for convenience we set $\Delta^{-v} f(t, a)=\Delta^{-v} f(t)$

(c) 2015 Akin et al. This article is distributed under the terms of the Creative Commons Attribution 4.0 International License (http://creativecommons.org/licenses/by/4.0/), which permits unrestricted use, distribution, and reproduction in any medium, provided you give appropriate credit to the original author(s) and the source, provide a link to the Creative Commons license, and indicate if changes were made. 
Theorem 1 [28] Let $f$ be a real valued function defined on $\mathbb{N}_{a}$ and let $\mu, v>0$. Then

$$
\Delta^{-\nu}\left(\Delta^{-\mu} f(t)\right)=\Delta^{-(\mu+\nu)} f(t)=\Delta^{-\mu}\left(\Delta^{-v} f(t)\right) \text { for all } t \in \mathbb{N}_{a+\mu+\nu} .
$$

Theorem 2 [21] For $v>0$ and $p$ a positive integer we have

$$
\Delta^{-v} \Delta^{p} f(t)=\Delta^{p} \Delta^{-v} f(t)-\sum_{k=0}^{v-1} \frac{(t-a)^{v-p+k}}{\Gamma(v+k-p+1)} \Delta^{k} f(a),
$$

where $f$ is defined on $\mathbb{N}_{a}$.

Remark 1 Let $\mu>0$ and $m-1<\mu<m, m=\lceil\mu\rceil$, where $m$ is a positive integer, and set $v=m-\mu>0$. Then by Theorem 2 we have

$$
\Delta^{-v} \Delta^{m} f(t)=\Delta^{m} \Delta^{-v} f(t)-\sum_{k=0}^{m-1} \frac{(t-a)^{\underline{v-m+k}}}{\Gamma(v+k-m+1)} \Delta^{k} f(a),
$$

where $f$ is defined on $\mathbb{N}_{a}$ and hence

$$
\Delta^{m} \Delta^{-v} f(t)=\Delta_{*}^{\mu} f(t)+\sum_{k=0}^{m-1} \frac{(t-a)^{\frac{v-m+k}{}}}{\Gamma(v+k-m+1)} \Delta^{k} f(a) .
$$

Definition 2 [21] The $\mu$ th fractional Riemann-Liouville type difference is defined by

$$
\Delta^{\mu} f(t):=\Delta^{m-v} f(t):=\Delta^{m}\left(\Delta^{-v} f(t)\right)
$$

where $\mu>0, m-1<\mu<m$, and $v=m-\mu>0$.

So from (2.1) we get

$$
\Delta^{\mu} f(t)=\Delta_{*}^{\mu} f(t)+\sum_{k=0}^{m-1} \frac{(t-a)^{\frac{v-m+k}{}}}{\Gamma(v+k-m+1)} \Delta^{k} f(a),
$$

where $f$ is defined on $\mathbb{N}_{a}$.

Theorem 3 [10] For $\mu>0, \mu$ noninteger, $m=\lceil\mu\rceil, v=m-\mu$, the following holds:

$$
f(t)=\sum_{k=0}^{m-1} \frac{(t-a)^{\underline{k}}}{k !} \Delta^{k} f(a)+\frac{1}{\Gamma(\mu)} \sum_{s=a+v}^{t-\mu}(t-s-1) \frac{\mu-1}{-} \Delta_{*}^{\mu} f(s)
$$

for all $t \in \mathbb{N}_{a+m}$, where $f$ is defined on $\mathbb{N}_{a}$ with $a \in \mathbb{Z}^{+}:=\{0,1,2, \ldots\}$.

Remark 2 Here $[a, b]$ denotes the discrete interval $[a, b]=[a, a+1, a+2, \ldots, b]$, where $a<b$ and $a, b \in\{0,1, \ldots\}$. Let $\mu>0$ be noninteger such that $m-1<\mu<m$, i.e. $m=\lceil\mu\rceil$. Consider a function $f$ defined on $[a, b]$. Then clearly the fractional discrete Taylor formula (2.3) is valid only for $t \in[a+m, b], a+m<b$. 
We now give a discrete Caputo type fractional extended Taylor formula.

Theorem 4 [10] Let $\mu>p, p \in \mathbb{N}, \mu$ not integer, $m=\lceil\mu\rceil, v=m-\mu$. Then

$$
\Delta^{p} f(t)=\sum_{k=p}^{m-1} \frac{(t-a) \frac{k-p}{(k-p) !}}{k} \Delta^{k} f(a)+\frac{1}{\Gamma(\mu-p)} \sum_{s=a+\nu}^{t-\mu+p}(t-s-1) \frac{\mu-p-1}{\Delta_{*}^{\mu}} f(s)
$$

for all $t \in \mathbb{N}_{a+m-p}$, where $f$ is defined on $\mathbb{N}_{a}, a \in \mathbb{Z}^{+}$.

Remark 3 We assume that $f$ is defined on $[a, b]$. Then (2.4) is valid only for $[a+m-p, b]$ with $a+m-p<b$. Notice $p=0$ applied to (2.4) yields (2.3).

Remark 4 For $\mu>0, \mu$ not an integer, $m=\lceil\mu\rceil, v=m-\mu, f$ defined on $\mathbb{N}_{a}, a \in \mathbb{Z}^{+}$and $\Delta^{k} f(a)$ for $k=0, \ldots, m-1$, we get

$$
f(t)=\frac{1}{\Gamma(\mu)} \sum_{s=a+v}^{t-\mu}(t-s-1)^{\mu-1} \Delta_{*}^{\mu} f(s) \quad \text { for all } t \in \mathbb{N}_{a+m}
$$

Remark 5 For $\mu>p, p \in \mathbb{N}, \mu$ noninteger, $m=\lceil\mu\rceil, v=m-\mu$; $f$ defined on $\mathbb{N}_{a}, a \in \mathbb{Z}^{+}$, if we assume that $\Delta^{k} f(a)=0, k=p, \ldots, m-1$, then we obtain

$$
\Delta^{p} f(t)=\frac{1}{\Gamma(\mu-p)} \sum_{s=a+\nu}^{t-\mu+p}(t-s-1) \frac{\mu-p-1}{} \Delta_{*}^{\mu} f(s) \quad \text { for all } t \in \mathbb{N}_{a+m-p} .
$$

\section{Main results}

We present the following discrete delta Grüss type inequality.

Theorem 5 Let $\mu>p, p \in \mathbb{Z}^{+}, \mu$ not an integer, $m=\lceil\mu\rceil, v=m-\mu, f, g$ be defined on $\mathbb{N}_{a}$, $a \in \mathbb{Z}^{+}$and $a+m-p<b, b \in \mathbb{N}$. Assume that

$$
\Delta^{k} f(a)=\Delta^{k} g(a)=0 \quad \text { for } k=p+1, \ldots, m-1, p<m-2,
$$

and

$$
m_{1} \leq \Delta_{*}^{\mu} f(s) \leq M_{1}, \quad m_{2} \leq \Delta_{*}^{\mu} g(s) \leq M_{2}
$$

for $s=a+1, \ldots, b$, where $m_{1}, m_{2}, M_{1}$, and $M_{2}$ are positive constants. Then

$$
\begin{aligned}
& \frac{1}{b-a-m+p} \sum_{j=a+m-p+1}^{b}\left[\left(\Delta^{p} f(j)\right)\left(\Delta^{p} g(j)\right)\right] \\
& \quad-\frac{1}{(b-a-m+p)^{2}}\left[\sum_{j=a+m-p+1}^{b} \Delta^{p} f(j)\right]\left[\sum_{j=a+m-p+1}^{b} \Delta^{p} g(j)\right] \\
& \leq \frac{M_{1} M_{2} C_{1}-m_{1} m_{2} C_{2}}{[\Gamma(\mu-p+1)]^{2}}
\end{aligned}
$$


where

$$
C_{1}:=(b-a-m+p) \sum_{j=a+m-p+1}^{b}\left[(j-a-v) \frac{\mu-p}{2}\right]^{2}
$$

and

$$
C_{2}:=\frac{\left[(b-a-v+1)^{\mu-p+1}-(m-p-v+1)^{\mu-p+1}\right]^{2}}{(\mu-p+1)^{2}} .
$$

Proof By (2.4), we have

$$
\Delta^{p} f(j)=\sum_{k=p}^{m-1} \frac{(j-a)^{\frac{k-p}{}}}{(k-p) !} \Delta^{k} f(a)+\frac{1}{\Gamma(\mu-p)} \sum_{s=a+\nu}^{j-\mu+p}(j-s-1) \frac{\mu-p-1}{} \Delta_{*}^{\mu} f(s) .
$$

By hypothesis $\Delta^{k} f(a)=0, k=p+1, \ldots, m-1, p<m-2$. So we have

$$
\Delta^{p} f(j)=\Delta^{p} f(a)+\frac{1}{\Gamma(\mu-p)} \sum_{s=a+v}^{j-\mu+p}(j-s-1) \frac{\mu-p-1}{2}\left(\Delta_{*}^{\mu} f(s)\right)
$$

and

$$
\Delta^{p} g(j)=\Delta^{p} g(a)+\frac{1}{\Gamma(\mu-p)} \sum_{s=a+\nu}^{j-\mu+p}(j-s-1) \frac{\mu-p-1}{2}\left(\Delta_{*}^{\mu} g(s)\right)
$$

for all $j \in[a+m-p+1, \ldots, b]$. Multiplying (3.1) and (3.2) gives us

$$
\begin{aligned}
\left(\Delta^{p} f(j)\right)\left(\Delta^{p} g(j)\right)= & \left(\Delta^{p} f(a)\right)\left(\Delta^{p} g(a)\right)+\frac{1}{[\Gamma(\mu-p)]^{2}} \\
& \times\left[\sum_{s=a+\nu}^{j-\mu+p}(j-s-1)^{\mu-p-1}\left(\Delta_{*}^{\mu} f(s)\right)\right]\left[\sum_{s=a+\nu}^{j-\mu+p}(j-s-1) \frac{\mu-p-1}{\left.\left(\Delta_{*}^{\mu} g(s)\right)\right]}\right. \\
& +\frac{\Delta^{p} f(a)}{\Gamma(\mu-p)}\left[\sum_{s=a+\nu}^{j-\mu+p}(j-s-1) \frac{\mu-p-1}{\left.\left(\Delta_{*}^{\mu} g(s)\right)\right]}\right. \\
& +\frac{\Delta^{p} g(a)}{\Gamma(\mu-p)}\left[\sum_{s=a+\nu}^{j-\mu+p}(j-s-1) \frac{\mu-p-1}{2}\left(\Delta_{*}^{\mu} f(s)\right)\right] .
\end{aligned}
$$

Summing from $a+m-p+1$ to $b$ yields

$$
\begin{aligned}
& \sum_{j=a+m-p+1}^{b}\left(\Delta^{p} f(j)\right)\left(\Delta^{p} g(j)\right) \\
& =\sum_{j=a+m-p+1}^{b}\left(\Delta^{p} f(a)\right)\left(\Delta^{p} g(a)\right)+\frac{1}{[\Gamma(\mu-p)]^{2}} \\
& \quad \times \sum_{j=a+m-p+1}^{b}\left\{\left[\sum_{s=a+\nu}^{j-\mu+p}(j-s-1)^{\frac{\mu-p-1}{2}}\left(\Delta_{*}^{\mu} f(s)\right)\right]\left[\sum_{s=a+\nu}^{j-\mu+p}(j-s-1) \frac{\mu-p-1}{(}\left(\Delta_{*}^{\mu} g(s)\right)\right]\right\}
\end{aligned}
$$




$$
\begin{aligned}
& +\frac{\Delta^{p} f(a)}{\Gamma(\mu-p)} \sum_{j=a+m-p+1}^{b}\left[\sum_{s=a+\nu}^{j-\mu+p}(j-s-1)^{\mu-p-1}\left(\Delta_{*}^{\mu} g(s)\right)\right] \\
& +\frac{\Delta^{p} g(a)}{\Gamma(\mu-p)} \sum_{j=a+m-p+1}^{b}\left[\sum_{s=a+\nu}^{j-\mu+p}(j-s-1)^{\mu-p-1}\left(\Delta_{*}^{\mu} f(s)\right)\right] .
\end{aligned}
$$

Then

$$
\begin{aligned}
& \frac{1}{b-a-m+p} \sum_{j=a+m-p+1}^{b}\left(\Delta^{p} f(j)\right)\left(\Delta^{p} g(j)\right) \\
& =\frac{1}{b-a-m+p} \sum_{j=a+m-p+1}^{b}\left[\left(\Delta^{p} f(a)\right)\left(\Delta^{p} g(a)\right)\right]+\frac{1}{(b-a-m+p)[\Gamma(\mu-p)]^{2}} \\
& \quad \times \sum_{j=a+m-p+1}^{b}\left\{\left[\sum_{s=a+\nu}^{j-\mu+p}(j-s-1) \frac{\mu-p-1}{2}\left(\Delta_{*}^{\mu} f(s)\right)\right]\left[\sum_{s=a+\nu}^{j-\mu+p}(j-s-1)^{\mu-p-1}\left(\Delta_{*}^{\mu} g(s)\right)\right]\right\} \\
& +\frac{\Delta^{p} f(a)}{(b-a-m+p) \Gamma(\mu-p)} \sum_{j=a+m-p+1}^{b}\left[\sum_{s=a+\nu}^{j-\mu+p}(j-s-1)^{\frac{\mu-p-1}{2}}\left(\Delta_{*}^{\mu} g(s)\right)\right] \\
& +\frac{\Delta^{p} g(a)}{(b-a-m+p) \Gamma(\mu-p)} \sum_{j=a+m-p+1}^{b}\left[\sum_{s=a+\nu}^{j-\mu+p}(j-s-1)^{\mu-p-1}\left(\Delta_{*}^{\mu} f(s)\right)\right] .
\end{aligned}
$$

On the other hand,

$$
\begin{aligned}
& \frac{1}{b-a-m+p} \sum_{j=a+m-p+1}^{b}\left(\Delta^{p} f(j)\right) \\
& =\Delta^{p} f(a)+\frac{1}{(b-a-m+p) \Gamma(\mu-p)} \sum_{j=a+m-p+1}^{b}\left[\sum_{s=a+\nu}^{j-\mu+p}(j-s-1) \frac{\mu-p-1}{-}\left(\Delta_{*}^{\mu} f(s)\right)\right]
\end{aligned}
$$

and

$$
\begin{aligned}
& \frac{1}{(b-a-m+p)} \sum_{j=a+m-p+1}^{b}\left(\Delta^{p} g(j)\right) \\
& \quad \Delta^{p} g(a)+\frac{1}{(b-a-m+p) \Gamma(\mu-p)} \sum_{j=a+m-p+1}^{b}\left[\sum_{s=a+\nu}^{j-\mu+p}(j-s-1)^{\mu-p-1}\left(\Delta_{*}^{\mu} g(s)\right)\right] .
\end{aligned}
$$

Multiplying the above two terms yields

$$
\begin{aligned}
& \frac{1}{(b-a-m+p)^{2}}\left[\sum_{j=a+m-p+1}^{b}\left(\Delta^{p} f(j)\right)\right]\left[\sum_{j=a+m-p+1}^{b}\left(\Delta^{p} g(j)\right)\right] \\
& =\left(\Delta^{p} f(a)\right)\left(\Delta^{p} g(a)\right) \\
& \quad+\frac{\Delta^{p} f(a)}{(b-a-m+p) \Gamma(\mu-p)} \sum_{j=a+m-p+1}^{b}\left[\sum_{s=a+\nu}^{j-\mu+p}(j-s-1)^{\mu-p-1}\left(\Delta_{*}^{\mu} g(s)\right)\right]
\end{aligned}
$$




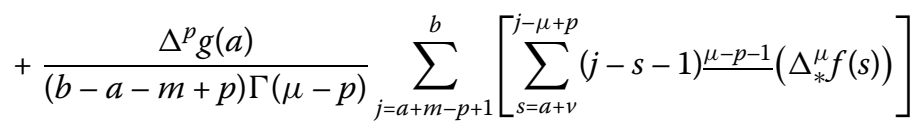

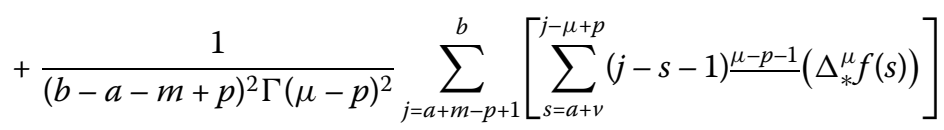

$$
\begin{aligned}
& \times \sum_{j=a+m-p+1}^{b}\left[\sum_{s=a+v}^{j-\mu+p}(j-s-1) \frac{\mu-p-1}{\left.\left(\Delta_{*}^{\mu} g(s)\right)\right] .}\right.
\end{aligned}
$$

So, using (3.3) and (3.4), we get

$$
\begin{aligned}
& \frac{1}{b-a-m+p} \sum_{j=a+m-p+1}^{b}\left[\left(\Delta^{p} f(j)\right)\left(\Delta^{p} g(j)\right)\right] \\
& \quad-\frac{1}{(b-a-m+p)^{2}}\left[\sum_{j=a+m-p+1}^{b}\left(\Delta^{p} f(j)\right)\right]\left[\sum_{j=a+m-p+1}^{b}\left(\Delta^{p} g(j)\right)\right] \\
& \leq \frac{M_{1} M_{2}}{(b-a-m+p)^{2}[\Gamma(\mu-p)]^{2}} \sum_{j=a+m-p+1}^{b}\left[\sum_{s=a+v}^{j-\mu+p}(j-s-1) \frac{\mu-p-1}{s}\right]^{2} \\
& \quad-\frac{m_{1} m_{2}}{(b-a-m+p)^{2}[\Gamma(\mu-p)]^{2}}\left[\sum_{j=a+m-p+1}^{b}\left[\sum_{s=a+v}^{j-\mu+p}(j-s-1) \frac{\mu-p-1}{2}\right]\right]^{2} .
\end{aligned}
$$

Now, calculating the sums:

$$
\sum_{s=a+\nu}^{j-\mu+p}(j-s-1) \frac{\mu-p-1}{=} \int_{\tau=a+\nu}^{j-\mu+p+1}(j-\sigma(\tau)) \frac{\mu-p-1}{\Delta \tau} \Delta=\frac{1}{\mu-p}(j-a-v) \frac{\mu-p}{,},
$$

We get

$$
\begin{aligned}
\sum_{j=a+m-p+1}^{b}\left[\sum_{s=a+v}^{j-\mu+p}(j-s-1) \frac{\mu-p-1}{2}\right]^{2} \leq & \frac{1}{(\mu-p)^{2}} \sum_{j=a+m-p+1}^{b}\left[(j-a-v) \frac{\mu-p}{2}\right]^{2}, \\
{\left[\sum_{j=a+m-p+1}^{b}\left[\sum_{s=a+v}^{j-\mu+p}(j-s-1) \frac{\mu-p-1}{2}\right]\right.} & =\frac{1}{(\mu-p)^{2}}\left[\sum_{j=a+m-p+1}^{b-a-v}(s) \frac{\mu-p}{2}\right]^{2} \\
= & \frac{1}{(\mu-p)^{2}} \frac{1}{(\mu-p+1)^{2}} \\
& \times[(b-a-v+1) \underline{\mu-p+1}-(m-p-v+1) \underline{\mu-p+1}]^{2}
\end{aligned}
$$

Consequently, we get

$$
\begin{aligned}
& \frac{1}{b-a-m+p} \sum_{j=a+m-p+1}^{b}\left[\left(\Delta^{p} f(j)\right)\left(\Delta^{p} g(j)\right)\right] \\
& \quad-\frac{1}{(b-a-m+p)^{2}}\left[\sum_{j=a+m-p+1}^{b}\left(\Delta^{p} f(j)\right)\right]\left[\sum_{j=a+m-p+1}^{b}\left(\Delta^{p} g(j)\right)\right]
\end{aligned}
$$




$$
\begin{aligned}
\leq & \frac{M_{1} M_{2}}{(b-a-m+p)[\Gamma(\mu-p)]^{2}} \frac{1}{(\mu-p)^{2}} \sum_{j=a+m-p+1}^{b}\left[(j-a-v) \frac{\mu-p}{-2}\right]^{2} \\
& -\frac{m_{1} m_{2}}{(b-a-m+p)^{2}[\Gamma(\mu-p)]^{2}} \frac{1}{(\mu-p)^{2}(\mu-p+1)^{2}} \\
& \times\left[(b-a-v+1) \frac{\mu-p+1}{\left.-(m-p-v+1) \frac{\mu-p+1}{2}\right]^{2}}\right. \\
= & \frac{M_{1} M_{2} C_{1}-m_{1} m_{2} C_{2}}{(b-a-m+p)^{2}[\Gamma(\mu-p+1)]^{2}} .
\end{aligned}
$$

\section{Competing interests}

The authors declare that they have no competing interests.

\section{Authors' contributions}

All authors contributed equally in writing this article and collaborated in its design in coordination. All authors read and approved the final paper.

\section{Author details}

${ }^{1}$ Department of Mathematics and Statistics, Missouri University S\&T, Rolla, MO, USA. ${ }^{2}$ Department of Mathematics, Faculty of Science, Ankara University, Ankara, Turkey. ${ }^{3}$ Department of Mathematics and Computer Science, Çankaya University, Ankara, 06810, Turkey.

\section{Acknowledgements}

Authors are grateful to the editor and reviewers for their suggestions.

Received: 31 October 2014 Accepted: 8 May 2015 Published online: 02 June 2015

\section{References}

1. Grüss, G: Über das Maximum des absoluten Betrages von $\frac{1}{b-a} \int_{a}^{b} f(x) g(x) d x-\frac{1}{(b-a)^{2}} \int_{a}^{b} f(x) d x \int_{a}^{b} g(x) d x$. Math. Z. 39 , 215-226 (1935)

2. Bohner, M, Peterson, A: Advances in Dynamic Equations on Time Scales. Birkhäuser, Boston (2003)

3. Cerone, P, Dragomir, SS: A refinement of the Grüss inequality and applications. Tamkang J. Math. 38, 37-49 (2007)

4. Dragomir, SS: A generalization of Grüss's inequality in inner product spaces and applications. J. Math. Anal. Appl. 237, 74-82 (1999)

5. Dragomir, SS: A Grüss type discrete inequality in inner product spaces and applications. J. Math. Anal. Appl. 250, 494-511 (2000)

6. Liu, Z: Notes on a Grüss type inequality and its applications. Vietnam J. Math. 35, 121-127 (2007)

7. Perić, I, Rajić, R: Grüss inequality for completely bounded maps. Linear Algebra Appl. 390, 287-292 (2004)

8. Pečarić, JE, Tepeš, B: On the Grüss type inequalities of Dragomir and Fedotov. J. Inequal. Pure Appl. Math. 4(5), 91 (2003)

9. Pečarić, JE, Tepeš, B: A note on Grüss type inequality in terms of $\Delta$-seminorms. Pril. - Maked. Akad. Nauk. Umet., Odd. Mat.-Teh. Nauki 23/24 (2002/2003); 29-35 (2004)

10. Anastassiou, GA: Nabla Discrete fractional calculus and inequalities. arXiv:0911.3374v1 [math.CA] (2009)

11. Anastassiou, GA: Multivariate Fink type identity and multivariate Ostrowski, comparison of means and Grüss type inequalities. Math. Comput. Model. 46, 351-374 (2007)

12. Bohner, M, Matthews, T: The Grüss inequality on time scales. Commun. Math. Anal. 3, 1-8 (2007) (electronic)

13. Graham, RL, Knuth, DE, Patashnik, O: Concrete Mathematics: A Foundation for Computer Science, 2nd edn. Addison-Wesley, Reading (1994)

14. Mercer, AM: An improvement of the Grüss inequality. JIPAM. J. Inequal. Pure Appl. Math. 6(4), 93 (2005)

15. Mitrinović, DS: Analytic Inequalities. Springer, New York (1970)

16. Mitrinović, DS, Pečarić, JE, Fink, AM: Classical and New Inequalities in Analysis. Kluwer Academic, Dordrecht (1993)

17. Pachpatte, BG: Some new Ostrowski and Grüss type inequalities. Tamkang J. Math. 38(2), 11-120 (2007)

18. Boros, G, Moll, V: Irresistible Integrals: Symbols, Analysis and Experiments in the Evaluation of Integrals. Cambridge University Press, Cambridge (2004)

19. Diaz, JB, Osler, TJ: Differences of fractional order. Math. Comput. 28, 185-202 (1974)

20. Gray, HL, Zhang, NF: On a new definition of fractional difference. Math. Comput. 50, 513-529 (1988)

21. Atıcı, FM, Eloe, PW: Initial value problems in discrete fractional calculus. Proc. Am. Math. Soc. 137(3), $981-989$ (2009)

22. Atıcl, FM, Sengül, S: Modeling with fractional difference equations. J. Math. Anal. Appl. 369(1), 1-9 (2010)

23. Goodrich, CS: Continuity of solutions to discrete fractional initial value problems. Comput. Math. Appl. 59(11), 3489-3499 (2010)

24. Atıcl, FM, Eloe, PW: Gronwall's inequality on discrete fractional calculus. Comput. Math. Appl. 64(10), 3193-3200 (2012)

25. Anastassiou, GA: Nabla discrete fractional calculus and nabla inequalities. Math. Comput. Model. 51(5-6), 562-571 (2010)

26. Güvenilir, AF, Kaymakçalan, B, Peterson, AC, Taş, K: Nabla discrete fractional Grüss type inequality. J. Inequal. Appl. 2014, 86 (2014). doi:10.1186/1029-242X-2014-86

27. Ferreira, RAC: A discrete fractional Gronwall inequality. Proc. Am. Math. Soc. 140(5), 1605-1612 (2012)

28. Atıcı, F, Eloe, P: A transform method in discrete fractional calculus. Int. J. Difference Equ. 2, 165-176 (2007) 